Case Report

\title{
Pediatric Chronic Myeloid Leukemia with Megakaryocytic Blast Crisis as Initial Presentation: Case Report and Review of Literature
}

\author{
Tuba Iqbal ${ }^{1}$, Amber Younus $^{1}$, Uzma Zaidi $^{*}$, Jawad Hassan², Tahir Sultan Shamsi ${ }^{1}$ \\ ${ }^{1}$ Department of Clinical Hematology, National Institute of Blood Disease \& Bone Marrow Transplantation, Karachi, Pakistan. \\ ${ }^{2}$ Department of Diagnostic Hematology, National Institute of Blood Disease \& Bone Marrow Transplantation, Karachi, Pakistan.
}

\begin{abstract}
Background: Pediatric Chronic Myeloid Leukemia (CML) is a rare entity accounting for 2-3\% of pediatric malignancies. CML rarely presents as Blast Crisis (BC) at the time of diagnosis, and megakaryocytic blast crisis is even rarer.

Case Presentation: We herein, report a case of a young female, 10-year-old who presented with anemia, leukocytosis and massive splenomegaly. Clinical features, peripheral film and bone marrow findings were consistent with CML in megakaryocytic blast crisis. Bone marrow cytogenetic analysis revealed karyotype of 46, XX, t(9:22)(q34;q11.2) in 20 metaphases and BCR-ABL P210 by PCR was detected with transcript level of $83 \%$, which further confirmed our diagnosis.
\end{abstract}

Conclusion: De novo presentation of chronic myeloid leukemia with megakaryocytic blast crisis is rarely observed in pediatric population with very few cases published till now. We are presenting this case because of its rarity, likelihood of misdiagnosis as AML (M7) and poor prognosis, if not treated precisely.

Keywords: Chronic Myeloid Leukemia (CML), Acute Myeloid Leukemia (AML), Blast Crisis (BC), Acute Megakaryocytic Leukemia (AMKL), Chronic Phase (CP), Accelerated Phase (AP), Tyrosine Kinase Inhibitor (TKI).

\section{INTRODUCTION}

Chronic Myeloid Leukemia (CML) is one of the clonal myeloproliferative hematopoietic stem cell disorders involving balanced reciprocal translocation between chromosome 9 and 22 [1]. CML typically occurs between fifth to sixth decade of life (age range $=17-68$ years). In Pakistan, CML occurs in relatively younger age group (median age is 38 years) [2]. CML naturally presents in 3 phases: Chronic phase (CP), accelerated phase (AP) and blast crisis (BC). Majority of the cases are diagnosed in chronic phase while less than $10 \%$ of the patients being diagnosed in advanced phase. Mostly Blasts are of myeloid in origin followed by lymphoid blast crisis that occurs in 1/3rd of cases and megakaryocytic origin in less than $3 \%$ of cases [1]. The frequency of the different phases of CML in Pakistani population at diagnosis was found to be $77.8 \%, 15.5 \%$ and $6.7 \%$ for $\mathrm{CP}, \mathrm{AP}$ and $\mathrm{BC}$ respectively, as reported by Riaz Ahmed et al. [2]. The prognosis of patients depends on the type of blast crisis, cytogenetic and the molecular findings of the patient [1]. Patients with CML-BC must be differentiated from de novo $\mathrm{Ph}+\mathrm{AML}$, as CML-BC can benefit from utilization of tyrosine kinase inhibitors (TKI) [3]. Here, we report a case of a young girl who presented to us in CML-BC with blast cells of megakaryocytic origin. We described this case due to its rarity and worse prognosis.

*Address correspondence to this author at the Department of Clinical Hematology, National Institute of Blood Disease \& Bone Marrow Transplantation, Karachi, Pakistan. Email: uzaidi26@gmail.com

\section{CASE REPORT}

A 10-year-old girl was referred to NIBD \& BMT in November 2018 with complaints of progressive abdominal distension along with constitutional B symptoms for the past 2 months. She had never been transfused. Personal or family history was unremarkable. Physical examination revealed mild pallor and splenomegaly (4 fingers below the left costal margin).

Complete blood count showed hemoglobin $(\mathrm{Hb}) 8.2 \mathrm{~g} / \mathrm{dl}$, white blood cells $($ WBC) $25.9 \times 109 / \mathrm{L}$ and platelet count $291 \times 109 / \mathrm{L}$. The peripheral smear showed poikilocytosis, tear drop cells, leuco erythroblastic picture, significant basophilia (6\%) and blast cells (39\%). The blasts were medium to large having intermediate $\mathrm{N} / \mathrm{C}$ ratio, irregular cellular margins, moderately basophilic agranular cytoplasm with distinct pseudopods formation and fine nuclear chromatin with prominent 2-3 nucleoli. Biochemical profile was normal. LDH was raised. Ultrasound abdomen showed splenomegaly $(16.6 \mathrm{~cm})$.

Bone marrow biopsy revealed cellularity of around $60-65 \%$. There was increased number of blast cells with prominent mitotic figures. Marked megakaryocytic proliferation was seen with megakaryocytes forming dense clusters and exhibiting marked dysplastic features such as monolobation and multinuclearity. Extensive background fibrosis was noted. Reticulin stain revealed grade II fibrosis corresponding to extensive intersections with focal bundles of collagen and focal osteosclerosis. (Fig. 1A, B).

www.njhsciences.com 


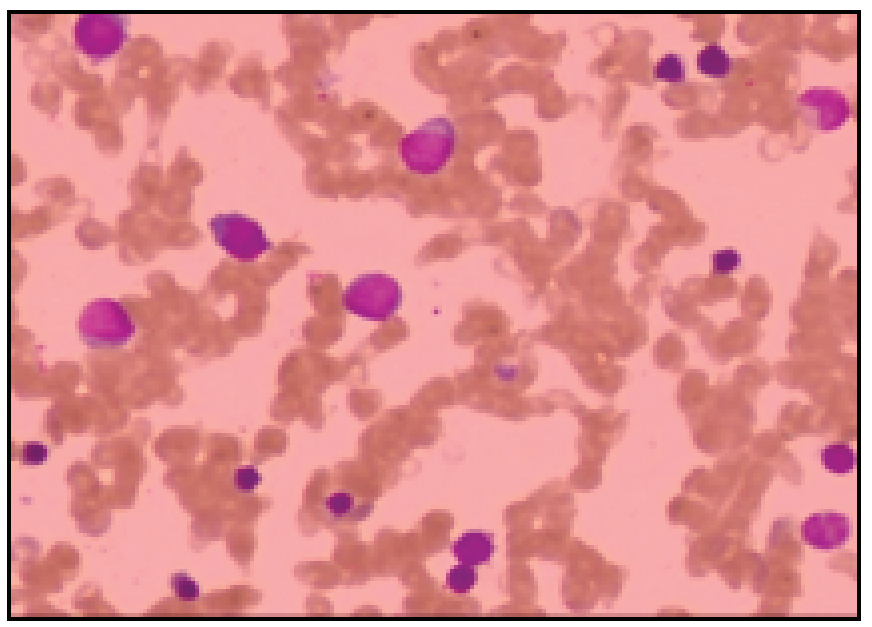

Fig. (1A). Bone Marrow Aspirate Showing Blast Cells.

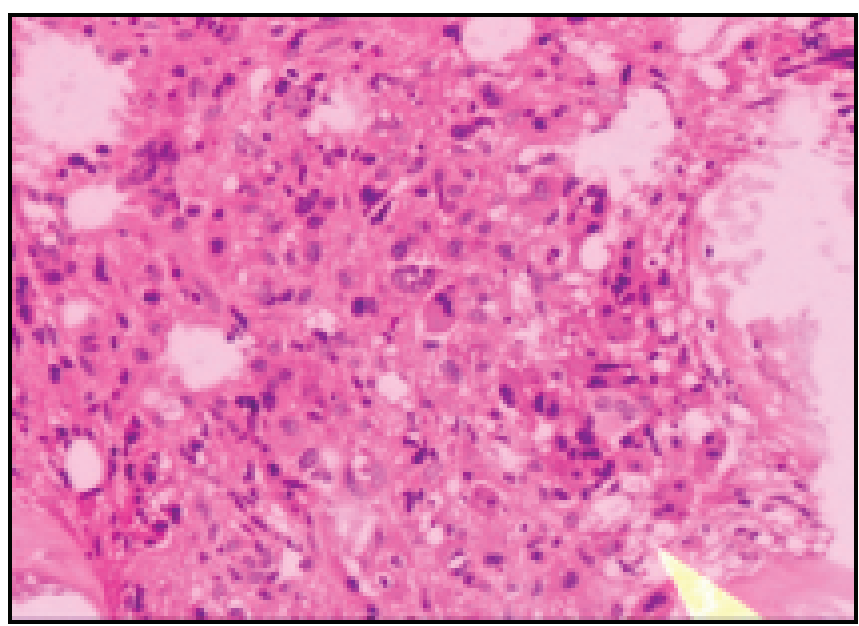

Fig. (1B). Trephine Biopsy Section Stained with H\&E Showing Megakaryocytic Proliferation \& Atypia with Extensive Background Fibrosis.

The results of immunohistochemistry performed on trephine sections demonstrated negativity in blast cells for CD34, TdT, CD117,CD20, CD79a, CD3 and MPO. Platelet specific marker CD 61 was strongly positive in blasts, PAS was inconclusive and glycophorin A was negative. (Fig. 2A, B). Her bone marrow cytogenetics showed karyotype of 46,XX with Philadelphia chromosome in all metaphases observed.

In summary, the clinical presentation along with presence of increased number of blasts that is $>20 \%$ in both peripheral blood and bone marrow exhibiting positivity for CD 61 and VWF along with extensive marrow fibrosis supported by Philadelphia chromosomes positivity on cytogenetic analysis compelled us to consider an uncommon evolution of CML blast crisis with megakaryocytic differentiation.

She was started with first-line tyrosine kinase inhibitor imatinib mesylate $400 \mathrm{mg}$ /day with hydroxyurea $500 \mathrm{mg}$ once daily for cytoreduction. Upon follow up in June 2019, she came with complaints of abdominal discomfort associated with constitutional B symptoms. On examination there was massive splenomegaly crossing the umbilicus. She has been counselled for chemotherapy along with TKI therapy followed by bone marrow transplant with HLA matched donor.

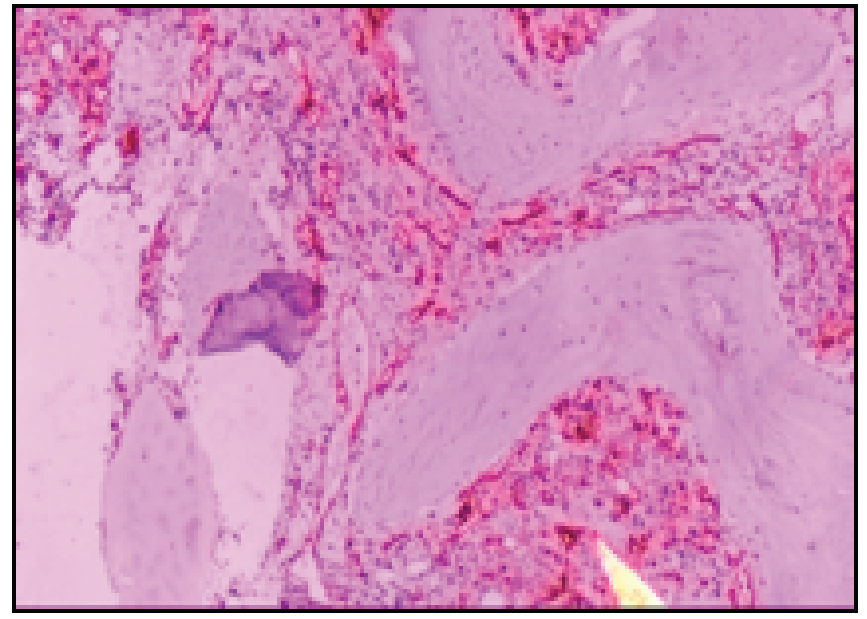

Fig. (2A). Immunohistochemistry Showing Negative Anti-CD34 in Blast Cells.

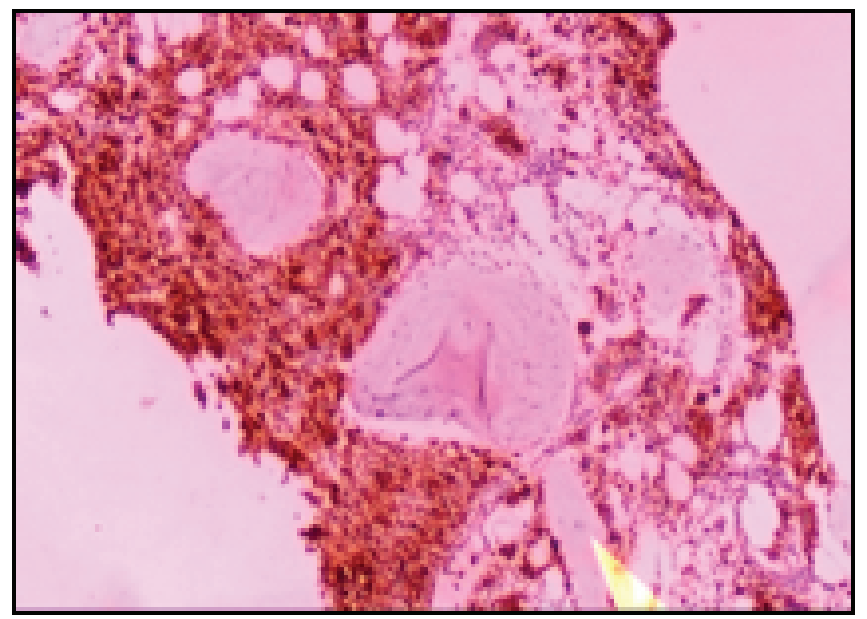

Fig. (2B). Positive Anti- CD61.

\section{DISCUSSION}

Data from literature on pediatric chronic myeloid leukemia (CML) represents $2-3 \%$ of leukemias in pediatric population aged younger than 15 years with median age of 11 years at presentation and has an incidence of 0.6-1.2/million children/year. It has an increased prevalence in males (1.2:1). Only $10 \%$ of children present in blast crisis (Suttorp \& Millot, 2010) [3]. Myeloid blast crisis is the most common presentation followed by lymphoid or mixed lineage type [4]. Among myeloid blast crisis, megakaryocytic type (AML M7) is the 
rarest entity accounting for less than $3 \%$ of cases [5]. There is very limited data regarding children with $\mathrm{CML}$ in advanced stages due to the small number of cases reported till date and having a tendency to present as more aggressive disease as compared to adults [6,7]. As compared to myeloid or lymphoid crisis, megakaryoblastic crisis has even a poorer prognosis [4]. Megakaryoblastic crisis is considered as a bad prognostic marker for relapse free survival and it's outcome depends on treatment strategy as it gives better results when treated with imatinib then proceeded by addition of chemotherapy in order to achieve chronic phase [4].

The differential diagnosis included de novo acute megakaryocytic leukemia, acute panmyelosis with myelofibrosis, transformed primary myelofibrosis and CML with blast crisis [8]. According to literature, thrombocytosis, splenomegaly along with basophilia may support the diagnosis of CML-BC [9]. All of these clinical features were present in our patient, favoring the diagnosis of CML rather than denovo AML. In CML BC, megakaryocytic lineage was confirmed by presence of definite megakaryocytic lineage markers, including CD41, CD42b, CD61, CD62 and Factor VIII [5]. In this case CD61 was strongly positive along with VWF. Philadelphia chromosome characteristically seen in CML has infrequently been described in AML, including AMKL.

It is important to distinguish de novo $\mathrm{AMKL}$ from $\mathrm{CML}$ megakaryocytic blast crisis because the therapeutic modalities and prognosis of both disorders vary significantly [10]. In contrast to Cytarabine-based induction regimen used for de novo AMKL, the addition of tyrosine kinase inhibitor along with induction chemotherapy regimen in CML blast crisis produce considerably better results.Initiating treatment with a tyrosine kinase inhibitor, with the aim of achieving chronic phase, can provide better results following stem cell transplantation [4]. In literature, poor outcome has been observed with AML directed chemotherapy alone with an average survival rate of 3.5-15 months in responders and 2-4 months in non-responders, whereas better outcome has been reported in patients treated with chemotherapy combined with imatinib [11]. In a pediatric case report, AML directed chemotherapy alone failed to achieve CHR after first induction, however, complete hematological response (CHR) and complete cytogenetic response (CCR) were achieved by subsequent addition of imatinib to second induction cycle, followed by major molecular response (MMR) prior to SCT [4]. In children with advanced-phase CML, allogenic SCT is considered as a preferable curative option over imatinib alone, once chronic phase is achieved and donor is identified [12]. Literature review suggested that treatment with a tyrosine kinase inhibitor may or may not combined with AML directed chemotherapy should be given and bone marrow transplantation should be planned as soon as possible [13] preferably within 3 months as suggested in NCCN guidelines $[3,14,15]$.
In this case, we started the patient on $1^{\text {st }}$ generation tyrosine kinase inhibitor, imatinib 400mg once daily along with cytoreduction with hydroxyurea 500mg per day. On follow up visit, she was asymptomatic but still have massive splenomegaly with peripheral blood basophilia and blast count of $27 \%$. We plan to give her cytarabine and anthracycline based AML-directed induction chemotherapy concomitantly with imatinib to improve outcome and to achieve chronic stable phase until we proceed to SCT depending on availability of HLA-matched donor.

\section{AUTHORS' CONTRIBUTION}

Author has confirmed that all authors have contributed equally.

\section{CONFLICT OF INTEREST}

Declared none.

\section{ACKNOWLEDGEMENTS}

Declared none.

\section{REFERENCES}

[1] Bhattacharya JB, Gupta R, Samadhiya A. Acute megakaryoblastic blast crisis as a presentation manifestation of chronic myelogenous leukemia. Blood Res 2017; 52(2): 137-9. DOI: $10.5045 /$ br.2017.52.2.137

[2] Ahmed R, Naqi N, Hussain I, Khattak BK, Nadeem M, Iqbal J. Presentating phases of chronic myeloid leukaemia. J Coll Physicians Surg Pak 2009; 19(8): 469-72.

[3] de la Fuente J, Baruchel A, Biondi A, et al. International BFM Group (iBFM) Study Group Chronic Myeloid Leukaemia Committee. Managing children with chronic myeloid leukaemia (CML) Recommendations for the management of CML in children and young people up to the age of 18 years. Br J Haematol 2014; 167(1): 33-47. DOI: 10.1111/bjh.12977

[4] Al-Shehri A, Al-Seraihy A, Owaidah TM, Belgaumi AF. Megakaryocytic blast crisis at presentation in a pediatric patient with chronic myeloid leukemia. Hematol Oncol Stem Cell Ther 2010; 3(1): 42-6.

DOI: $10.1016 / \mathrm{S} 1658-3876(10) 50056-6$

[5] Pagano L, Pulsoni A, Vignetti M, et al. Acute megakaryoblastic leukemia: Experience of GIMEMA trials. Leukemia 2002; 16(9): 1622-6. DOI: 10.1038/sj.leu.2402618

[6] Hijiya N, Schultz KR, Metzler M, Millot F, Suttorp M. Pediatric chronic myeloid leukemia is a unique disease that requires a different approach. Blood 2016; 127(4): 392-9. DOI: 10.1182/blood-2015-06-648667

[7] Millot F, Guilhot J, Suttorp M, et al. Advanced phases at diagnosis of childhood chronic myeloid leukemia: The experi- 
ence of the international registry for chronic myeloid leukemia (CML) in children and adolescents (I-CML-Ped Study). Blood 2017; 130(Suppl 1): 316.

[8] Ashar KM, Vaghasiya V, Patel SC. Megakaryocytic blast crisis in chronic myeloid leukemia: A primary presentation. Indian J Pathol Microbiol 2017; 60(3): 445. DOI: 10.4103/IJPM.IJPM_841_16

[9] Soupir CP, Vergilio JA, Cin PD, et al. Philadelphia chromosome-positive acute myeloid leukemia: A rare aggressive leukemia with clinicopathologic features distinct from chronic myeloid leukemia in myeloid blast crisis. Am J Clin Pathol 2007; 127(4): 642-50. DOI: 10.1309/B4NVER1AJJ84CTUU

[10] Jarmuż M, Kroll R, Przybyłowicz-Chalecka A, et al. Megakaryocytic blast crisis in a chronic myeloid leukemia patient with a rare variant of Philadelphia rearrangement $t(9 ; 22 ; 22)$ and a constitutional translocation $\mathrm{t}(3 ; 7)$. Cancer Genet Cytogenet 2010; 199(1): 45-7.

DOI: $10.1016 /$ j.cancergencyto.2010.01.017
[11] Morris EL, Dutcher JP. Blastic phase of chronic myelogenous leukemia. Clin Adv Hematol Oncol 2005; 3(7): 547-52.

DOI: $10.1007 / \mathrm{s} 11864-006-0012-y$

[12] Suttorp M. Innovative approaches of targeted therapy for CML of childhood in combination with paediatric haematopoietic SCT. Bone Marrow Transplant 2008; 42(2): S40-6. DOI: $10.1038 / \mathrm{bmt} .2008 .282$

[13] Hehlmann R. How I treat CML blast crisis. Blood 2012; 120(4): 737-47. DOI: 10.1182/blood-2012-03-380147

[14] Shulman DS, Lee MA, Lehmann LE, Margossian SP. Outcomes following bone marrow transplantation in children with accelerated phase or blast crisis chronic myelogenous leukemia in the era of tyrosine kinase inhibitors. J Pediatr Hematol Oncol 2016; 38(8): 610-4.

DOI: 10.1097/MPH.0000000000000636

[15] Hehlmann R, Saussele S, Voskanyan A, Silver RT. Management of CML-blast crisis. Best Pract Res Clin Haematol 2016; 29(3): 295-307. DOI: 10.1016/j.beha.2016.10.005 